\title{
Development, characterization, antioxidant and hepatoprotective properties of poly $(\epsilon$-caprolactone) nanoparticles loaded with a neuroprotective fraction of Hypericum perforatum
}

\author{
Ana Isabel Oliveira ${ }^{\mathrm{a}, * *}$, Cláudia Pinho $^{\mathrm{a}}$, Pedro Fonte ${ }^{\mathrm{b}, \mathrm{c}}$, Bruno Sarmento $^{\mathrm{d}, \mathrm{e}, \mathrm{f}}$, \\ Alberto C.P. Dias ${ }^{g, *}$ \\ a Nucleo de Investigação e Informação em Farmácia (NIIF), Centro de Investigação em Saúde e Ambiente (CISA), Escola Superior de Saúde -Politécnico do \\ Porto (ESS-P.Porto), 4000-072 Porto, Portugal \\ ${ }^{\mathrm{b}}$ UCIBIO, REQUIMTE, Department of Chemical Sciences - Applied Chemistry Lab, Faculty of Pharmacy, University of Porto, $4050-113$ Porto, Portugal \\ ${ }^{\mathrm{C}}$ CBIOS, Universidade Lusófona Research Center for Biosciences \&'Health Technologies, Campo Grande 376, 1749-024 Lisboa, Portugal \\ d i3S - Instituto de Investigação e Inovação em Saúde, Universidade do Porto, Rua Alfredo Allen 208, 4200-135 Porto, Portugal \\ e INEB - Instituto Nacional de Engenharia Biomédica, Universidade do Porto, Rua Alfredo Allen 208, 4200-135 Porto, Portugal \\ ${ }^{\mathrm{f}}$ CESPU, Instituto de Investigação e Formação Avançada em Ciências e Tecnologias da Saúde and Instituto Universitário de Ciências da Saúde, $4585-116$ \\ Gandra, Portugal \\ $\mathrm{g}$ Centre for the Research and Technology of Agro-Environment and Biological Sciences (CITAB-UM), AgroBioPlant Group, Department of Biology, University \\ of Minho, 4710-057 Braga, Portugal
}

\section{A R T I C L E I N F O}

\section{Article history:}

Received 20 June 2017

Received in revised form 4 October 2017

Accepted 16 October 2017

Available online $\mathrm{xxx}$

\section{Keywords:}

Hypericum perforatum

Quercetin

Biapigenin

Nanoparticles

Anti-oxidant

Hepatoprotective

In vitro release

\begin{abstract}
A B S T R A C T
Quercetin and biapigenin are antioxidant and neuroprotective compounds present in Hypericum perforatum, with potential application in neurodegenerative diseases. Quercetin has shown to have excellent antioxidant activities. Biapigenin possesses a distinctive mechanism of action, preventing the onset of calcium deregulation and mitochondrial dysfunction. The main aim of this study was to establish a new delivery system encapsulating $H$. perforatum neuroprotective fraction, isolated from it,containing quercetin and biapigenin into poly $(\epsilon$-caprolactone) $(\mathrm{PCL})$ nanoparticles. It also aimed to study its hepatoprotective potential. Different formulation parameters were optimized, resulting in a stable formulation of polymer:compounds ratio 1:0.1 with a mean particle size of $185 \mathrm{~nm}$, zeta potential around $-20 \mathrm{mV}$ and association efficiency close to $100 \%$. Compounds in vitro release, under physiological conditions, showed an initial burst followed by a sustained release. The antioxidant functional properties of these compounds were not altered by encapsulation. PCL-loaded nanoparticles protected $\mathrm{HepG}_{2}$ cells from intrinsic compound toxicity at high concentrations. Depending on the incubation regimen, quercetinbiapigenin PCL-loaded nanoparticles or free compounds were more effective in protecting $\mathrm{HepG}_{2}$ cells against tert-butylhydroperoxide-induced toxicity. This is the first report of the encapsulation of a quercetin-biapigenin mixture in a polymeric matrix, specifically, in PCL, with synergic anti-oxidant and hepatoprotective effects.
\end{abstract}

(c) 2017 Elsevier B.V. All rights reserved.

\section{Introduction}

The central nervous system (CNS) is known being particularly sensitive to oxidative stress, which can be described as an

\footnotetext{
* Corresponding author at: Agrobioplant Group (CITAB-UM), Biology Department, University of Minho - Campus de Gualtar, 4710-057 Braga, Portugal.

** Corresponding author at: Escola Superior de Saúde, Politécnico do Porto, Rua António Bernardino de Almeida, 400, 4200-072 Porto, Portugal.

E-mail addresses: aio@ess.ipp.pt (A.I. Oliveira), acpdias@bio.uminho.pt (A.C.P. Dias).
}

https://doi.org/10.1016/j.ijbiomac.2017.10.103

0141-8130/@ 2017 Elsevier B.V. All rights reserved. imbalance between generation and elimination of reactive oxygen species (ROS) and reactive nitrogen species (RNS).

By-products of cells' energy production, ROS and RNS, result from the cellular redox process. These species play a dual effect either as toxic and/or beneficial compounds. At low or moderate levels, ROS and RNS exert beneficial effects on cellular responses and immune function [1]. At high concentrations, a disturbance in the pro-oxidant/antioxidant balance in favor of the pro-oxidant state may occur, which can lead to cell damage. Oxidative damage is, therefore, associated with aging and the development of several diseases such as cardiovascular and neurodegenerative disorders, cancer and diabetes, resulting in the loss of membrane integrity, structural and functional changes in proteins and gene mutations. 
ROS and RNS generated during the body's metabolic reactions can react with some cellular molecules, such as lipids, proteins and DNA, damaging them $[1,2]$.

It is therefore important to preserve redox environment and mitochondrial function of the cell. This can be achieved by avoiding the causes of oxidative stress and strengthen the defenses with the usage of endogenous antioxidants and the intake exogenous antioxidants [3]. Besides their usage in the prevention of neurodegenerative diseases, antioxidants could also be relevant on its treatment, as a single compound or in supplementary combination with drugs targeting other pathogenic mechanisms [4].

$H$. perforatum has been used as a medicinal plant for centuries, for the treatment of external and internal disorders, such as minor burns, wounds, skin inflammation, nerve pain, anxiety and mild to moderately severe depression, competing for status as a standard antidepressant therapy and being a valid herbal alternative to synthetic antidepressants [5].

$H$. perforatum extracts and compounds also showed to have relevant antioxidant and neuroprotective properties [2,6-9]. Specifically, a purified fraction containing quercetin and biapigenin has been extensively studied regarding its antioxidant and neuroprotective properties [6-9]. Quercetin has been described to protect against several oxidative insults and several types of ROS [8],and is considered the most potent scavenger of ROS, RNS and peroxynitrite of the flavonoid family [10]. Its ability to interact with multiple cellular targets is likely the basis of its pharmacological activity [11]. In vivo quercetin activity remains controversial. Quercetin presents low aqueous solubility and instability in physiological medium [12], depending on temperature and $\mathrm{pH}$, which limits its bioavailability, reduces permeability, and suffers extensive first pass metabolism before reaching the local of action [13]. Biapigenin is a less studied compound, possessing a distinctive mechanism of action, compared to quercetin. It was shown to prevent the onset of calcium deregulation and mitochondrial dysfunction, affecting mitochondrial bioenergetics [6,8,9]. Antioxidant activities of biapigenin have also been reported through its lipid peroxidation inhibition potential $[6,7]$.

One alternative to circumvent bioavailability problems is to entrap or adsorb the compounds into nanoparticles. The advantages of using nanoparticles for drug delivery result from their small size, which allows them to penetrate within small capillaries and be taken up within the cells, with efficient drug accumulation at the target sites in the body. It also allows sustained drug release within the target site over a period of days or even weeks after administration. [14]. Nanosystems employed for CNS targeted drug delivery include polymeric nanoparticles that compared with other colloidal carriers, are a relevant alternative. PCL is a semi-crystalline hydrophobic polymer, degraded by hydrolysis of its ester linkages under physiological conditions. Its high permeability to many drugs and a lack of toxicity has made PCL and its derivates well suited for drug delivery [15].

Liver is essential for survival because of its role in the coordination of the body's metabolism, including glucose homeostasis, xenobiotic metabolism and detoxification. All organs are vulnerable to oxidative stress. However, because of the high metabolic activity of hepatocytes, liver is highly susceptible to oxidative stress and a major target of toxic substances. Biotransformation of certain xenobiotics can also produce short-lived, unstable, highly reactive chemical species, such as ROS and RNS that can interact with functional biomolecules and lead to adverse effects, resulting in inflammatory and fibrotic liver disorders [16].

Human hepatocellular carcinoma cells $\left(\mathrm{HepG}_{2}\right)$ is a widely used human in vitro model for hepatic toxicity studies, including antioxidants that can be assayed with minor variations [16]. HepG ${ }_{2}$ cells retain many of the specialized functions characteristic of normal human hepatocytes, including the synthesis and secretion of plasma proteins, phase I, phase II and antioxidant enzymes, being good tool to study hepatic toxicology, cytoprotective, and antigenotoxic effects of compounds [17].

The main aim of this study was to establish a nanoparticulate system for the use of a neuroprotective fraction of $H$. perforatum containing quercetin and biapigenin, using the synthetic polymer PCL, by solvent displacement technique. The physical and morphological parameters of the nanoparticles were investigated, as well as its in vitro release profile. Furthermore, the antioxidant activities of the compounds and of its nanoparticles were determined. The potential protective effect of quercetin-biapigenin and its PCLloaded nanoparticles against $t$-BOOH-induced oxidative stress in $\mathrm{HepG}_{2}$ cells was also studied.

\section{Material and methods}

\subsection{Materials}

Dulbecco's Modified Eagle Medium (DMEM), antibioticantimycotic solution, glutamine, trypsin-ethylenedi aminetetraacetic acid (EDTA) solution, 3-(4,5-dimethy lthiazole-2-yl)-2,5diphenyl tetrazolium bromide (MTT), PCL, Pluronic ${ }^{\circledR}$ F-68, DPPH, nitroblue tetrazolium (NBT), reduced form of nicotinamide adenine dinucleotide (NADH), N-phenylmethazonium methosulfate (PMS), quercetin, biapigenin, curcumin, ferrozine, trichloroacetic acid and tiobarbituric acid were purchased from Sigma-Aldrich Chemicals Co. (St. Louis, MO, USA). Diethyl ether and acetone were purchased from Merck (Germany). Sephadex LH-20 and polyamide CC6 acquired were from GE Healthcare Life Sciences (UK). L(+)-Ascorbic acid and pyruvate were purchased from Panreac(Barcelona, Spain). EDTA was purchased from VWR (Portugal). Fetal Bovine Serum (FBS) was purchased from Biochrom KG (Berlin, Germany). All other reagents used were for analytical grade.

\subsection{Methods}

\subsubsection{Isolation of a neuroprotective Hypericum perforatum} fraction containing quercetin and biapigenin

$H$. perforatum plants (aerial portion) were collected in the region of Braga, North of Portugal, and the biomass was freeze-dried on the same day, in a Labconco lyophilizer at $0.01 \mathrm{mBar}$ for 4 days, with a condenser surface temperature of $-90^{\circ} \mathrm{C}$.

A methanolic extract was prepared by macerating plant biomass $(100 \mathrm{~g} \mathrm{dwb} / \mathrm{L})$ with a methanol-water solution $(80: 20, \mathrm{~V} / \mathrm{V})$, for 4 days, at room temperature and in darkness. The mixture was filtered through a paper filter (Whatman, No. 1) and the resulting methanolic extract was used for further processing. The acidified extract ( $\mathrm{pH} 3$ ) was fractionated by liquid-liquid extraction with diethyl ether and the resulting fraction concentrated, under reduced pressure, at $35^{\circ} \mathrm{C}$, in darkness. This ether fraction was further subjected to Sephadex LH-20 column chromatography, eluted with methanol. A selected fraction containing quercetin and biapigenin was collected, concentrated, and purified through Polyamide CC6 column. Compounds identification was performed by HPLCDAD as previously described [18]. Chromatograms were recorded at 260, 350 and $590 \mathrm{~nm}$, and quercetin and biapigenin were quantified $(350 \mathrm{~nm})$ by the external method, using pure commercial standards. This fraction is composed solely by quercetin and biapigenin and will be referred wherein as quercetin-biapigenin.

\subsubsection{Production of quercetin-biapigenin poly-(€-caprolactone) $(P C L)$ nanoparticles}

Different formulations of quercetin-biapigenin loaded PCL nanoparticles (PCL: quercetin-biapigenin ratio of $1: 0.1 ; 1: 0.2$ and 1:0.5) were prepared by nanoprecipitation, as described elsewhere [19]. Briefly, the corresponding mass of quercetin-biapigenin and 
$2.5 \mathrm{mg}$ of polymer were dissolved in $1 \mathrm{~mL}$ of acetone, transferred, drop by drop, into $10 \mathrm{~mL}$ of a Pluronic ${ }^{\circledR}$ F-68 aqueous solution and stirred at $550 \mathrm{rpm}$ for $3 \mathrm{~h}$. After that, nanoparticle suspension was centrifuged at $15000 \mathrm{rpm}$ for $30 \mathrm{~min}$ at $4{ }^{\circ} \mathrm{C}$ and supernatant discharged. Nanoparticles were washed with MilliQwater, lyophilized and stored in a moisture free environment for further use. Freeze-drying conditions were of $0.01 \mathrm{mBar}$, for 3 days, with a condenser surface temperature of $-90^{\circ} \mathrm{C}$.

\subsubsection{Mean particle size and zeta potential analysis}

The mean particle size and polydispersity index (PI) of the nanoparticles were determined by Dynamic Light Scaterring (DLS) using a Zetasizer NanoZS (Malvern Instruments, Ltd, UK). Zeta potential determination was performed by laser doppler microelectrophoresis, with the same equipment. The samples were diluted 10-times with MilliQ-water for analysis. Each sample was measured by three independent measurements, with three replica each, and the results are shown as mean \pm standard deviation.

\subsubsection{Association efficiency, loading capacity and production yield}

For the association efficiency (AE) determination, nanoparticles suspension was centrifuged, the supernatant was collected and analyzed by HPLC-DAD, as previously described [18]. The amount of quercetin and biapigenin encapsulated was determined by the following formula:

$$
\begin{gathered}
\mathrm{AE}(\%)=[\text { (Total amount of compounds }- \text { Free compounds } \\
\text { in supernatant }) / \text { Total amount of compounds }] \times 100
\end{gathered}
$$

Loading capacity (LC) was determined by the difference between the initial amount of compounds used to prepare the nanoparticles and the amount present in the supernatant after centrifugation. Total weight of nanoparticles was determined by lyophilization of an aliquot of hydrated nanoparticles.

$$
\begin{gathered}
\mathrm{LC}(\%)=[(\text { Total amount of compounds }- \text { Free compounds } \\
\text { in supernatant }) / \text { Total weight of nanoparticles }] \times 100
\end{gathered}
$$

Nanoparticles yield was determined taking in consideration the final weight of the nanoparticles (after lyophilization) and the initial amount of compounds to be encapsulated and polymer used in its preparation.

$$
\begin{aligned}
& \text { Yield }(\%)=\text { (Total weight of nanoparticles/Total weight of } \\
& \text { compounds and polymer fed initially }) \times 100
\end{aligned}
$$

Each sample was analyzed as three independent sets, with three replica each. The results are shown as mean \pm standard deviation.

\subsubsection{Nanoparticle morphology by transmission electronic microscopy \\ Morphologic analysis of the nanoparticles was performed by transmission electronic microscopy (TEM), using a TEM Zeiss model $902 \mathrm{C}, 80 \mathrm{KV}$. TEM samples were stained with uranyl acetate $1 \%$ solu- tion, and placed on copper grids. The excess of water was removed using a filter paper, and grids were left at room temperature for one minute to dry prior to imaging.}

\subsubsection{Fourier transform infrared spectroscopy (FTIR) spectroscopy}

Quercetin-biapigenin loaded PCL nanoparticles and unloaded PCL nanoparticles, were analyzed by FTIR spectroscopy to assess drug-polymer interactions upon encapsulation. Control samples of quercetin-biapigenin and its physical mixture with PCL polymer, were also collected. All FTIR spectra were collected from 100 scans, in the $4000-600 \mathrm{~cm}^{-1}$ range at $4 \mathrm{~cm}^{-1}$ resolution, on a ABB MB3000 FTIR (ABB, USA). After data collection, all spectra were normalized for further comparison.

\subsubsection{In vitro release profile}

Quercetin-biapigenin loaded PCL nanoparticles were dispersed in phosphate buffered saline (PBS) (pH 7.4) containing ethanol $10 \%$ $(\mathrm{V} / \mathrm{V})$ and incubated at $37^{\circ} \mathrm{C}$ under magnetic stirring at $100 \mathrm{rpm}$. Samples were taken at predetermined time intervals of $0.5,1,2$, 4,8 and $24 \mathrm{~h}$. The collected samples were centrifuged and the quercetin and biapigenin content in the supernatant was determined by HPLC. All samples were run in three independent sets, each composed by three replica, and the results are shown as mean \pm standard deviation.

\subsubsection{In vitro antioxidant activity}

2.2.8.1. 2,2-diphenyl-1-picrylhydrazyl (DPPH)-radical scavenging activity assay. The free radical-scavenger activity was determined by the DPPH assay, as described previously [7]. Briefly, after adding different concentrations of the free compounds and quercetin-biapigenin loaded PCL nanoparticles to DPPH $(90 \mu \mathrm{M})$, the percentage of remaining DPPH was determined continuously from the absorbance at $515 \mathrm{~nm}$ using a plate reader spectrophotometer, until a plateau state. At this time the percentage of remaining DPPH was plotted against the concentration of the compounds/loaded-PCL nanoparticles and the amount of antioxidant necessary to decrease by $50 \%$ the initial DPPH concentration (EC50) was calculated, using Graph Pad Prism 5.0 ${ }^{\circledR}$ software. Quercetin was used as positive control. All measurements were performed in three independent sets, each composed by three replica, and results are shown as mean \pm standard deviation.

2.2.8.2. Evaluation of superoxide radical scavenging activity. The superoxide radical scavenging activity was determined using the PMS-NADH nonenzymatic assay as previously described [20]. The reaction mixture in the sample wells consisted of NADH $(166 \mu \mathrm{M})$, NBT $(43 \mu \mathrm{M})$, free compounds and quercetin-biapigenin loaded PCL nanoparticles and PMS $(2.7 \mu \mathrm{M})$, in a final volume of $300 \mu \mathrm{L}$. All components were dissolved in phosphate buffer (19 mM, pH 7.4). The assays were performed at room temperature during $2 \mathrm{~min}$ and started with the addition of PMS. Ascorbic acid was used as positive control. The sample concentration providing 50\% inhibition (EC50) was obtained by plotting the inhibition percentage against extract concentrations, using Graph Pad Prism 5.0 ${ }^{\circledR}$ software. All measurements were performed in three independent sets, each composed by three replica and results are shown as mean \pm standard deviation.

2.2.8.3. Iron (II) chelating activity. The iron chelating activity of the free compounds and quercetin-biapigenin loaded PCL nanoparticles was evaluated as described previously [21] with some modifications. Briefly, $50 \mu \mathrm{L}$ of free compounds and PCL-loaded nanoparticles at different concentrations were added to a solution of $0.12 \mathrm{mM}$ of $\mathrm{FeSO}_{4}(50 \mu \mathrm{L})$ and $50 \mu \mathrm{L}$ of Ferrozine $(0.6 \mathrm{mM})$. Then the mixture was shaken vigorously and left standing at room temperature for $10 \mathrm{~min}$. Absorbance of the solution was then measured spectrophotometrically at $562 \mathrm{~nm}$. EDTA was used as a positive control. The ability of the free compounds and quercetin-biapigenin loaded PCL nanoparticles to chelate ferrous ion was calculated relative to the control using the formula:

Chelatingactivity $(\%)=($ Abscontrol - Abssample $) /$ Abscontrol $\times 100$ 
The sample concentration that chelates $50 \%$ of the ferrous ion (EC50) was calculated, using Graph Pad Prism 5.0 ${ }^{\circledR}$ software. All measurements were performed in three independent sets, each composed by three replica and results are shown as mean \pm standard deviation.

\subsection{Cell culture}

$\mathrm{HepG}_{2}$ cell line was obtained from the American Type Culture Collection (ATCC) and cultured in $25 \mathrm{~cm}^{2}$ flasks with DMEM, supplemented with 10\% FBS, 2 mM L-glutamine and 1\% antibioticantimycotic solution, under an atmosphere of $5 \% \mathrm{CO}_{2}$ at $37^{\circ} \mathrm{C}$. Cells were sub-cultured once a week using $0.25 \%$ trypsin-EDTA and seeded at a density of $2 \times 10^{6}$ cells per $\mathrm{mL}$.

\subsection{Protective effect of quercetin-biapigenin and} quercetin-biapigenin poly( $\epsilon$-caprolactone)-loaded nanoparticles assays against tert-butylhydroperoxide-induced toxicity

$\mathrm{HepG}_{2}$ cells were plated in 96-multiwell culture plates at a concentration of $2 \times 10^{5}$ cells per $\mathrm{mL}$.

To study the compounds and nanoparticle influence in cell viability, $40 \mathrm{~h}$ after plating, several concentrations of compounds or nanoparticles were added (concentration equivalent to $1-100 \mu \mathrm{g} / \mathrm{mL}$ of free compounds) and $24 \mathrm{~h}$ later metabolic activity of the cells was estimated by the MTT assay. Results were expressed as percentage of cell metabolic activity from the control (cells without any compound).

To study the compounds ability to protect $\mathrm{HepG}_{2}$ cells against $t$-BOOH-induced toxicity two incubation regimens were used: preincubation and co-incubation regimen, as described previously [22], with minor modifications. Briefly, in the pre-incubation regimen, cells were incubated with the compounds in a free state or with the quercetin and biapigenin PCL-loaded nanoparticles for $4 \mathrm{~h}$, followed by a recovery period of $16 \mathrm{~h}$ with fresh medium without the compounds to allow cells antioxidant defenses to be synthesized. Then cells were incubated with $1.5 \mathrm{mM}$ t-BOOH for $3.5 \mathrm{~h}$. In the co-incubation regimen $\mathrm{HepG}_{2}$ cells were incubated with the compounds or nanoparticles and $1.5 \mathrm{mM}$ t-BOOH for $3.5 \mathrm{~h}$ and $5 \mathrm{~h}$.

\subsection{Evaluation of quercetin-biapigenin mixture and} quercetin-biapigenin poly( $\epsilon$-caprolactone)-loaded nanoparticles against tert-butylhydroperoxide-induced lipid peroxidation

In order to study the potential protective effect of the compounds in its free form and in nanoparticles against $t-\mathrm{BOOH}-$ induced lipid peroxidation co-incubation regimen was used. Cells were plated in 6-multiwell plates at a concentration of $7.5 \times 10^{5}$ cells per mL. After $40 \mathrm{~h}$, cells were incubated with the compounds, quercetin-biapigenin PCL-loaded nanoparticles and/or $t$-BOOH for 3.5 h. After that cell medium was harvested and kept at $-80^{\circ} \mathrm{C}$ for further processing.

The extent of lipid peroxidation was estimated by the levels of MDA measured, using tiobarbituric and reactive substances (TBARS) assay, as previously described [20] with some modifications. Briefly, trichloroacetic acid 50\% (w/V) was added to the harvested medium and then the mixture was centrifuged at $10000 \mathrm{rpm}$ for $1 \mathrm{~min}$. After, tiobarbituric acid 1\% (w/V) was added to the supernatant. This was left for $10 \mathrm{~min}$ in a boiling water bath. After cooling, the solution was read at $535 \mathrm{~nm}$.

\subsection{Statistical analysis}

In most of the experiments three independent sets, each composed by three replicas, have been done. Statistical analysis was

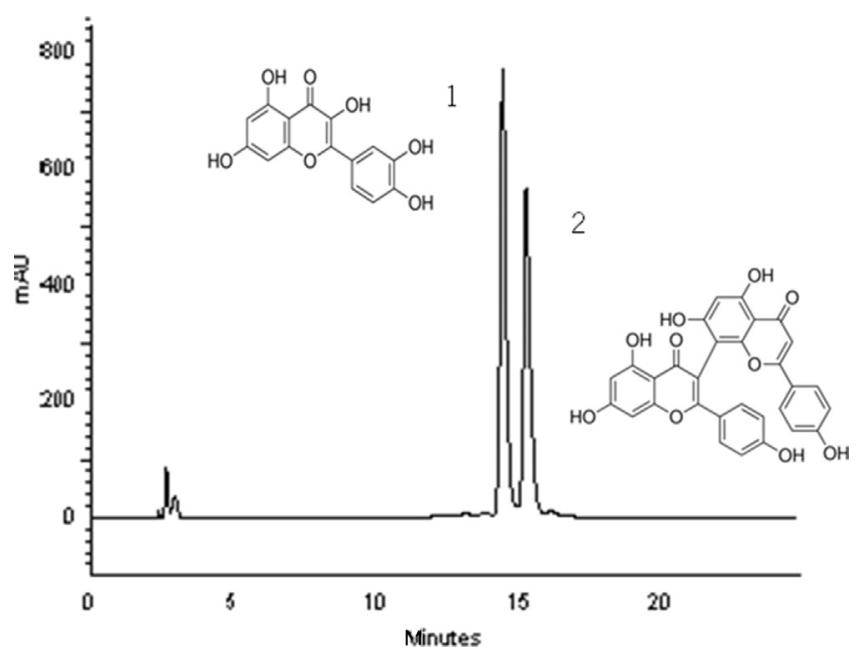

Fig. 1. Chromatographic profile of the compound mixture obtained; peak 1-quercetin; peak 2-biapigenin.

performed using Graph Pad Prism 5.0 ${ }^{\circledR}$ software. In the nanoparticles characterization, one-way ANOVA was employed with Tukey's post hoc multiple comparison test. In the in vitro antioxidant assays unpaired Students $t$-test was used. In the in vitro cell assays Oneway ANOVA was employed with Dunnett's multiple comparison test, when comparing each concentration against a control and unpaired $t$-test when comparing two similar concentrations. Differences were considered to be significant at a level of $p<0.05$.

\section{Results and discussion}

\subsection{Production of quercetin-biapigenin poly ( $\epsilon$-caprolactone) $(P C L)$ nanoparticles}

Through the isolation procedure described in material and methods a purified $H$. perforatum fraction, containing quercetin and biapigenin ( $1: 1 \mathrm{w} / \mathrm{w})$ as quantified by HPLC-DAD) (Fig. 1), was obtained. This fraction, proved to have relevant antioxidant and neuroprotective activities [6-9]. Quercetin-biapigenin loaded PCL nanoparticles were prepared by nanoprecipitation, also known as solvent displacement method. It is considered one of the easiest and mostly used method to obtain nanoparticles, especially with PCL polymer [15,19]. Additionally, it is a reproducible, fast and economic procedure that uses pre-formed polymers as starting materials. The polymer solvent (acetone) was chosen among organic solvents that are miscible in water and easy to remove by evaporation. For this reason, acetone is the most frequently used polymer solvent [23]. The nanoparticles form instantaneously during the rapid diffusion of the compound mixture-polymer solution in water. The use of a surfactant is not critical to nanoparticle formation [23]. Despite that, it helps to preserve nanoparticle suspension from aggregation, being therefore relevant for the physical stability of the preparation [24]. In this preparation the option was to use a well described poloxamer composed of (propylene oxide)/poly(ethylene oxide) block copolymer, Pluronic ${ }^{8}$ F-68. Nanoparticle preparation was found to be reproducible for size and zeta potential.

\subsection{Characterization of nanoparticles}

Particle size and size distribution are considered important characteristics of nanoparticulate systems, since they determine in vivo distribution, toxicity and its targeting ability. They can also influence drug loading, drug release and nanoparticle stability [25]. 
Table 1

Mean particle size, polydispersity, zeta potential, association efficiency and loading capacity of quercetin-biapigenin poly( $\epsilon$-caprolactone)-loaded nanoparticles.

\begin{tabular}{|c|c|c|c|c|c|c|c|c|}
\hline $\begin{array}{l}\text { PCL: } \\
\text { Compounds }\end{array}$ & Size (nm) & $\begin{array}{l}\text { Polydispersity } \\
\text { Index }\end{array}$ & $\begin{array}{l}\text { Zeta } \\
\text { potential } \\
(\mathrm{mV})\end{array}$ & $\begin{array}{l}\text { Association } \\
\text { Efficiency (Q) } \\
(\%)\end{array}$ & $\begin{array}{l}\text { Association } \\
\text { Efficiency (B) } \\
(\%)\end{array}$ & $\begin{array}{l}\text { Association } \\
\text { Efficiency } \\
(Q+B)(\%)\end{array}$ & $\begin{array}{l}\text { Loading } \\
\text { Capacity (\%) }\end{array}$ & $\begin{array}{l}\text { Nanoparticle } \\
\text { Yield (\%) }\end{array}$ \\
\hline $1: 0.1$ & $185 \pm 4$ & $0.11 \pm 0.03$ & $-20.1 \pm 1.2$ & $99.3 \pm 0.1$ & $99.9 \pm 0.1$ & $99.7 \pm 0.1$ & $5.3 \pm 0.1$ & $60.2 \pm 1.5$ \\
\hline $1: 0.2$ & $219 \pm 21^{\dagger}$ & $0.27 \pm 0.10$ & $-19.3 \pm 1.2$ & $54.3 \pm 14.6^{\dagger}$ & $67.5 \pm 11.5^{\dagger}$ & $67.3 \pm 14.4^{\dagger}$ & $9.2 \pm 1.8$ & $50.6 \pm 8.1$ \\
\hline $1: 0.5$ & $250 \pm 22^{\#, ~}$ & $0.37 \pm 0.04^{\#, \ddagger}$ & $-18.0 \pm 0.1^{\dagger}$ & $56.0 \pm 8.6^{\dagger}$ & $73.2 \pm 4.4^{\dagger}$ & $65.0 \pm 3.4^{\dagger}$ & $33.5 \pm 13.0^{\dagger, ¥}$ & $52.0 \pm 6.7$ \\
\hline
\end{tabular}

Q- quercetin, B- biapigenin; $\mathrm{n}=3$; results are showed as mean \pm standard deviation.

\# Significantly different compared with 1:0.1 $(p<0.001)$.

† Significantly different compared with 1:0.1 $(p<0.01)$.

‡ Significantly different compared with 1:0.2 $(p<0.01)$.

\& Significantly different compared with 1:0.1 $(p<0.001)$.

$¥$ Significantly different compared with 1:0.2 $(p<0.05)$.

As Table 1 shows, the mean particle size of quercetin-biapigenin loaded PCL nanoparticles were about $200 \mathrm{~nm}$, which was expected, taking into account the preparation method [24]. PI reveals a narrow distribution [25] and values under 0.4. The mean particle size and PI increases with the increase of theoretical loading capacity. This leads to more compound interaction and being the amount of polymer constant in all proportions, there could be expected a decrease in the ability of PCL to fully encapsulate all compounds in the colloidal suspension. In fact, the best proportion, in terms of size and stability is the one with less compounds to be encapsulated, 1:0.1 (mean particle size of $184.9 \pm 9.1 \mathrm{~nm}$ and PI of $0.11 \pm 0.03$ ).

Zeta potential values of the three proportions tested had a negative value, which may be attributed to the ionized carboxyl groups of PCL segments on the nanoparticles surface [26]. As for an absolute value, zeta potential was around $-20 \mathrm{mV}$, a good indicative of the nanoparticle stability [15].

Association efficiency (AE) and loading capacity (LC) are known to depend on the drug solubility in the polymer, which is related to polymer composition, molecular weight, drug polymer interaction and the presence of end functional groups (ester or carboxyl) [27]. In the present study, the nanoparticle $\mathrm{AE}$ was observed to be inversely proportional to compounds concentration. The highest $\mathrm{AE}$ was obtained in 1:0.1 PCL:compounds ratio. A higher LC was however expected in this proportion. Taking only in consideration AE percentage, one could estimate a LC close to $9 \%$. Taking into account the yield $(60.2 \pm 1.5 \%)$ it can be observed that LC is about $60 \%$ of the one expected for an $\mathrm{AE}$ of almost $100 \%$. These results are, thereby, complementary. Taking into consideration all these results the best stable formulation had a polymer:compounds ratio of 1:0.1.

\subsection{Nanoparticle morphology by transmission electronic microscopy (TEM)}

TEM morphological analysis of quercetin-biapigenin loaded PCL nanoparticles and empty PCL nanoparticles revealed that all were formed by spherical nanoparticles (Fig. 2A-D). Ratio 1:0.1 is the
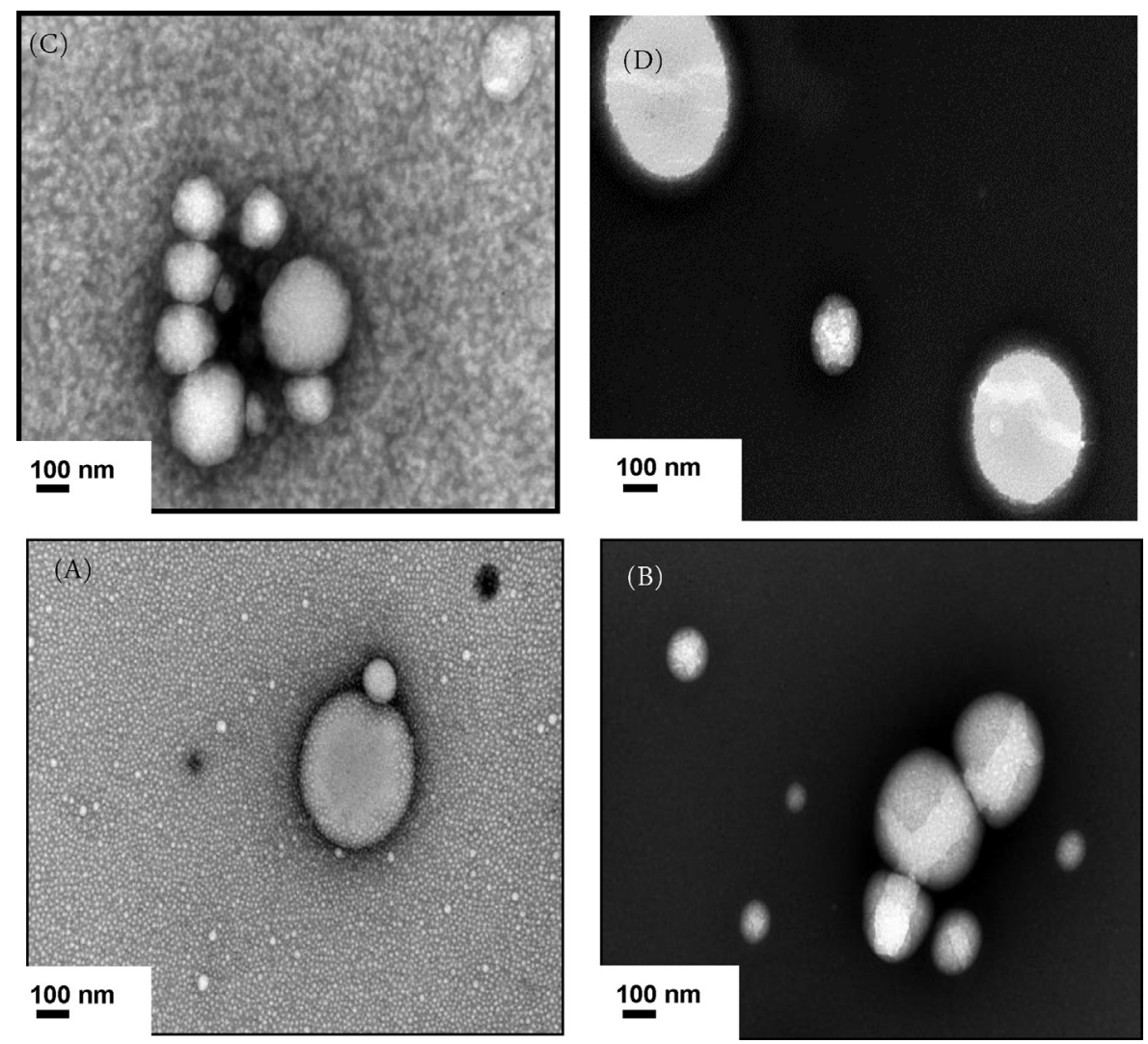

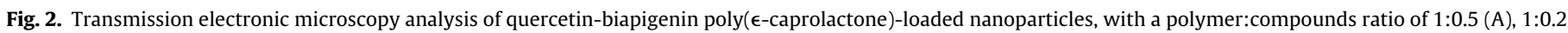
(B), 1:0.1 (C) and empty nanoparticles (D) at a total amplification of 100000X. 
।
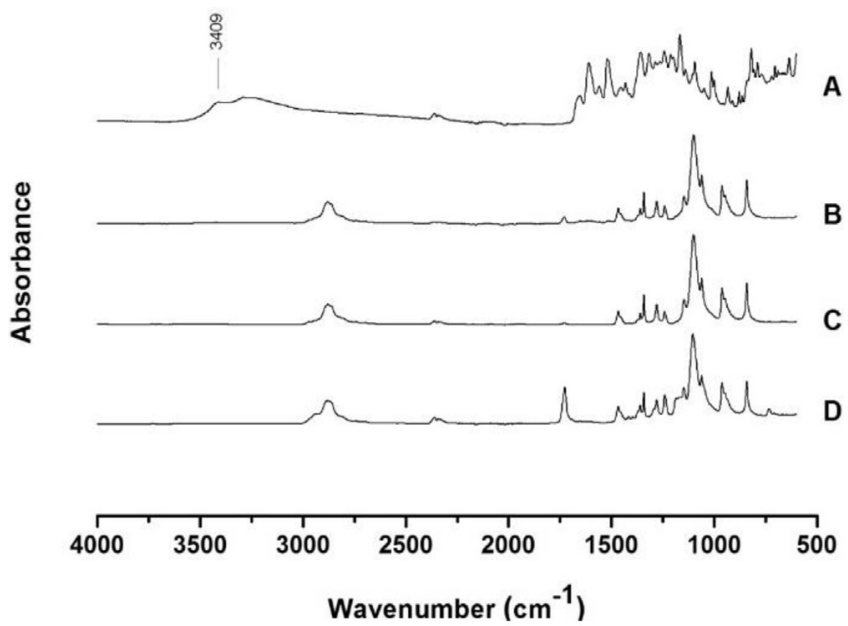

II
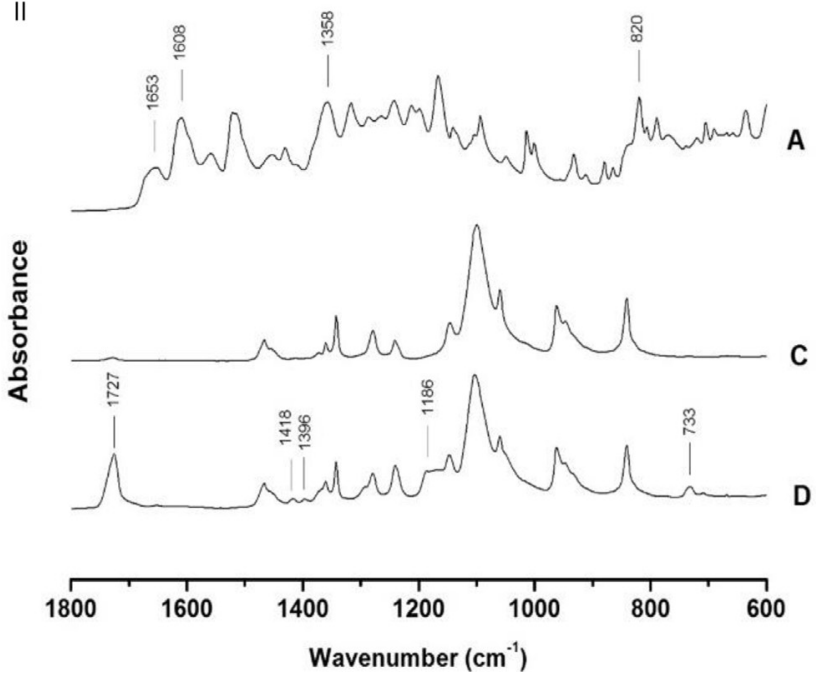

Fig. 3. FTIR spectra of quercetin-biapigenin (A), physical mixture of quercetinbiapigenin and PCL polymer (B), unloaded PCL nanoparticles (C) and quercetinbiapigenin loaded PCL nanoparticles (D) (I). FTIR spectra was truncated in the range of $1800-600 \mathrm{~cm}-1$, which represents the fingerprint region of the compounds (II).

one that presents smaller nanoparticles, with the most uniform size distribution of the three. Quercetin-biapigenin PCL-loaded nanoparticles TEM results are coherent with studies described in literature for PCL-loaded nanoparticles [27]. The TEM data of our study are consistent with the particle size measures.

\subsection{Fourier transform infrared spectroscopy (FTIR) spectroscopy}

FTIR is a very useful technique to assess the encapsulation of drugs and its intermolecular interactions in a nanoparticle system. The functional groups present in quercetin and biapigenin are similar so it is expected to obtain similar absorption bands in the same regions of those functional groups.

In Fig. 3 it is showed that quercetin-biapigenin presented an absorption band of $\mathrm{O}-\mathrm{H}$ stretch at $3409 \mathrm{~cm}^{-1}$ (Fig. 3-I) which is characteristic of various phenolic compounds, and a characteristic absorption band of $\mathrm{C}=\mathrm{O}$ at $1653 \mathrm{~cm}^{-1}$ (Fig. 3-II). The band at $1608 \mathrm{~cm}^{-1}$ is assigned to $C=C$ stretching vibrations occurred in the aromatic rings, and the band at $1358 \mathrm{~cm}^{-1}$ is characteristic of $\mathrm{O}-\mathrm{H}$ bending vibrations (Fig. 3-B). Furthermore, the strong absorption band at $820 \mathrm{~cm}^{-1}$ is assigned to the aromatic rings. The FTIR spectra of the physical mixture of quercetin-biapigenin and PCL and the

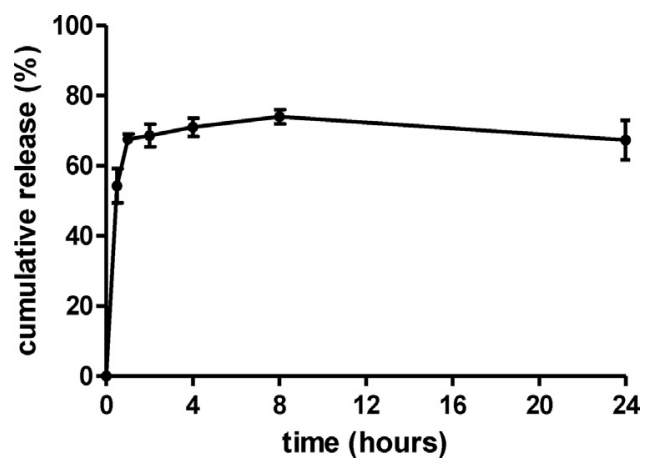

Fig. 4. Cumulative release profile of biapigenin from poly( $\epsilon$-caprolactone)-loaded nanoparticles in phosphate-buffered saline (PBS, pH 7.4) containing ethanol $10 \%$ $(\mathrm{V} / \mathrm{V})$. Data is presented as mean $\pm \mathrm{SD}$ of three independent experiments.

unloaded PCL nanoparticles were very similar (Fig. 3-II). The proportion of quercetin-biapigenin and PCL in the physical mixture was the same as used to produce the loaded PCL nanoparticles, and that may be the explanation why the spectra was so similar to the unloaded PCL nanoparticles spectra because the signal of PCL is much higher than that of quercetin-biapigenin. Nevertheless, it is possible to conclude that no intermolecular interactions occurred in the mixture since no significant bands occurred in the spectra.

The FTIR spectra of quercetin-biapigenin loaded PCL nanoparticles shows up the intermolecular interaction between quercetinbiapigenin and PCL polymer upon encapsulation. This was shown by the appearance of 1186 and $733 \mathrm{~cm}^{-1}$ bands that occurred in quercetin-biapigenin loaded PCL nanoparticles and did not occurred in the physical mixture neither in the unloaded PCL nanoparticles spectra. In addition, that interactions within the nanoparticle system was mainly showed by a great increase of the intensity of $1727 \mathrm{~cm}^{-1}$ absorption band. The latter is motivated by an increase of a $\mathrm{C}=\mathrm{O}$ stretching within the system, and the 1186 and $733 \mathrm{~cm}^{-1}$ bands may occurred due to OC-O stretching and an aromatic $\mathrm{C}-\mathrm{H}$ bending, respectively. Other small absorption bands occurred at 1418 and $1396 \mathrm{~cm}^{-1}$, which is characteristic of $\mathrm{C}-\mathrm{H}$ bending. Considering all the FTIR spectra and particularly the spectra of quercetin-biapigenin loaded PCL nanoparticles it is possible to conclude that quercetin-biapigenin drug was successfully entrapped into the PCL nanoparticles. That can also be concluded taking into account the similarity between empty and quercetinbiapigenin nanoparticles and its difference to the physical mixture.

\subsection{In vitro release profile}

Drug release and subsequent biodegradation are important features for developing successful formulations. The release rates of nanoparticles depend upon desorption of the surfacebound/adsorbed drug, diffusion through the nanoparticle matrix, nanoparticle matrix erosion or a combined erosion/diffusion process [28].

As shown in Fig. 4, there was as initial burst release of about $68 \%$ in the first hour. The burst release of hydrophobic compounds, such as quercetin and biapigenin, is attributed to the predominant surface presence of the compounds in the nanoparticle formulation [29]. After the first hour a sustained and slow release continued reaching $75 \%$ after $8 \mathrm{~h}$. Until the end of the assay, at $24 \mathrm{~h}$ time, there was not a significant alteration of the amount of compounds released. This can be explained by the predominant diffusion release mechanism rather than polymer degradation verified, especially with low molecular weight compounds, such as those in study [27].

As for the release medium in which the assay was performed, there had to be taken in consideration solubility aspects. The com- 
Table 2

Antioxidant activity (EC50) of free purified fraction and quercetin-biapigenin poly( $\epsilon$-caprolactone)-loaded nanoparticles.

\begin{tabular}{|c|c|c|c|}
\hline & \multicolumn{3}{|l|}{$\mathrm{EC} 50(\mu \mathrm{g} / \mathrm{mL})$} \\
\hline & $\begin{array}{l}\text { DPPH radical } \\
\text { scavenging activity }\end{array}$ & $\begin{array}{l}\text { Superoxide radical } \\
\text { scavenging activity }\end{array}$ & $\begin{array}{l}\text { Iron (II) chelating } \\
\text { activity }\end{array}$ \\
\hline Quercetin-biapigenin & $5.95 \pm 0.97$ & $72.71 \pm 4.07$ & $11.56 \pm 0.44$ \\
\hline Quercetin-biapigenin PCL-loaded nanoparticles & $5.73 \pm 1.20$ & n.d. & $23.50 \pm 0.55$ \# \\
\hline
\end{tabular}

n.d. - not detected the concentration range tested $(5-250 \mu \mathrm{g} / \mathrm{mL})$

\# Significantly different compared with quercetin-biapigenin $(p<0.001)$.

pounds in study are sparingly soluble in PBS, so ethanol was added to the buffer $(10 \%, \mathrm{~V} / \mathrm{V})$. Ethanol increased compounds solubility into the release medium [30], without affecting the release profile, since PCL is not soluble in it [31]. Release medium properties were maintained as physiologic as possible.

Fig. 4 only reports to the release profile of biapigenin, since quercetin, present in the initial mixture, was degraded due to PBS conditions. Quercetin instability in physiologic medium (half-life in PBS pH 7.2 of $10 \mathrm{~h} \mathrm{[32])} \mathrm{can} \mathrm{be} \mathrm{due} \mathrm{to} \mathrm{medium} \mathrm{alkalinization,}$ leading to oxidation by atmospheric oxygen [33] or by an autooxidative phenomenon that can occur in aerobic aqueous medium [34]. Despite that and due to the chemical similarity between quercetin and biapigenin (as perceived by the chemical structures in Fig. 1), one could expect a similar release profile for both compounds.

\subsection{In vitro antioxidant activity}

\subsubsection{2,2-diphenyl-1-picrylhydrazyl (DPPH)-radical scavenging activity assay}

DPPH method is a decolorization assay which measures the relative antiradicalar abilities of natural compounds and extracts to scavenge free radicals. It is a commonly used method to measure antioxidant activity due to excellent reproducibility, stability, commercial availability and by the fact that it is an easy screening/measurement method [7]. The antiradicalar activities of the free and nanoencapsulated compounds are shown in Table 2. Quercetin is known to be a good DPPH radical scavenger, with low EC50 values reported, whereas biapigenin is not a good DPPH scavenger [47]. In our study EC50 of compound mixture, quercetin and biapigenin, was of $5.95 \pm 0.97 \mu \mathrm{g} / \mathrm{mL}$, which can be essentially attributed to quercetin's activity (Table 2). This antiradicalar activity was lower, in comparison to positive control, quercetin $(1.85 \pm 0.18 \mu \mathrm{g} / \mathrm{mL})$, which can be explained by the presence of biapigenin in the mixture. Compounds in its free form and in PCL-loaded nanoparticles had similar antiradicalar activities. This result was consistent with other reports for quercetin nanoparticles [29,35].

\subsubsection{Evaluation of superoxide radical scavenging activity}

Superoxide radical is formed in almost all aerobic cells as a result of the donation of one electron of oxygen and is a major agent in the mechanism of oxygen toxicity. Itself is a week radical but may cause severe damage to the cell by generating hydroxyl radical and singlet oxygen [36].

The scavenging activity of free purified fraction and loaded PCL nanoparticles was established by the NBT reduction method, where the non-enzymatic PMS/NADH system generates superoxide radicals, which reduce the yellow $\mathrm{NBT}^{2+}$ in order to produce the purple NBT formazan. Antioxidants are able to inhibit the purple NBT formation by competition with NBT for superoxide radical [36].

The quercetin-biapigenin mixture showed a good superoxide radical scavenging activity $(E C 50=77.64 \pm 9.46 \mu \mathrm{g} / \mathrm{mL}$ ) (Table 2), similar to the positive control, ascorbic acid $(E C 50=77.50 \pm 10.88 \mu \mathrm{g} / \mathrm{mL})$. Superoxide scavenging activity is related to the same structural molecular features as of DPPH scavenging activity [37], and likewise, in our study, attributed to quercetin. In quercetin-biapigenin loaded PCL nanoparticles, despite of an increase of activity verified with the increase of concentration tested, there was not possible to determine an EC50 value, in concentration range tested. The time in which the assay takes place (three min, as described in the material and methods section) and according to the release profile of these nanoparticles, will not be enough to allow the release of quercetin-biapigenin from the polymeric matrix. These results are coherent with the compound encapsulation in PCL matrix.

\subsubsection{Iron (II) chelating activity}

Iron can stimulate lipid peroxidation by the Fenton reaction and by decomposing lipid hydroperoxides into peroxyl and alkoxyl radicals that can perpetuate chain of reaction. Metal chelating capacity is therefore significant since it reduces the concentration of the transition metal that catalyzes lipid peroxidation. Ferrozine produces a violet complex with $\mathrm{Fe}^{2+}$. In the presence of a chelating agent, complex formation is interrupted and as a result the violet color of the complex is decreased [38].

Regarding iron chelation (IC), the compounds mixture presented a good activity (Table 2), despite lower than EDTA $(\mathrm{EC} 50=1.18 \pm 0.01 \mu \mathrm{g} / \mathrm{mL}$ ). Iron (II) chelating activity of the mixture can be attributed to quercetin, which is known to be powerful chelating agent that can sequester $\mathrm{Fe}^{2+}$ in such a way to prevent its involvement in the Fenton reaction [38]. It can possibly also be due to biapigenin if we take in consideration previous reports that attributed IC a possible role in the inhibition of lipid peroxidation of compounds such as apigenin, amentoflavone and biapigenin [6,39]. In quercetin-biapigenin loaded PCL nanoparticles the IC value was significantly higher $(p<0.001)$. This, can be explained by the methodology employed. There is a $10 \mathrm{~min}$ incubation period previously to the IC measurement. This will allow the release of some amount of quercetin and biapigenin from the nanoparticle, but not enough for a complete release of the compounds, resulting in a lower IC activity. These results are coherent with the compound encapsulation in PCL matrix.

\subsection{Long-term toxicity of compounds and nanoparticles}

Since liver is the organ primary responsible for compound metabolization and due to its susceptibility to toxic effects, its relevant to determine the toxicity/protective activity of quercetin and biapigenin, in its free form and in PCL-loaded nanoparticles.

In order to select appropriate concentrations for the cytoprotective assays, non-toxic concentrations were first determined, ensuring that no direct cell damage was caused by compounds tested.

The compounds tested, encapsulated or not, showed increasing cytotoxicity in a concentration dependent manner (Fig. 5). In concentrations higher than $25 \mu \mathrm{g} / \mathrm{mL}$, for non-encapsulated quercetin and biapigenin $(p<0.05)$, and $50 \mu \mathrm{g} / \mathrm{mL}$, for quercetin-biapigenin PCL-loaded nanoparticles $(p<0.01)$, there was significant toxicity on $\mathrm{HepG}_{2}$ cells. 


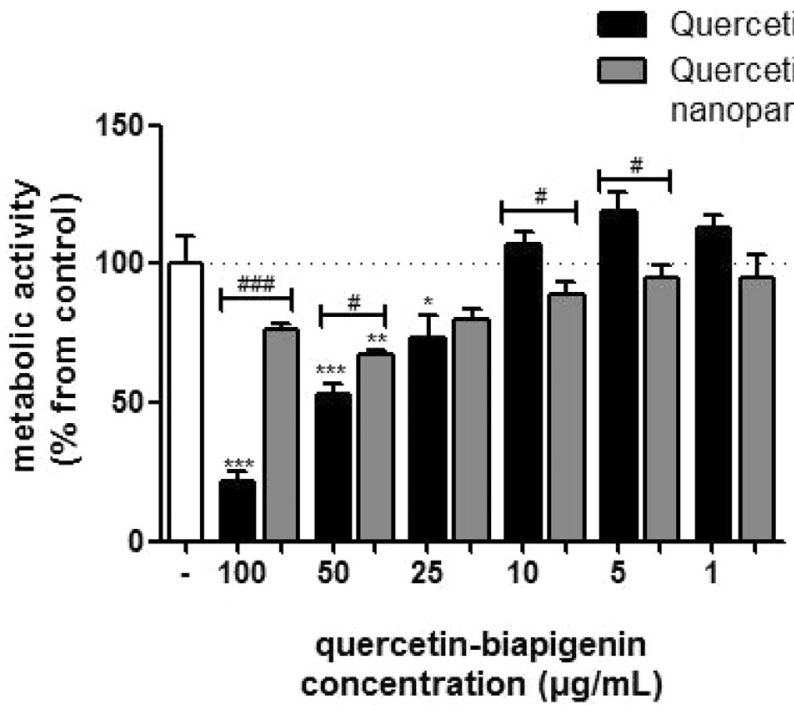

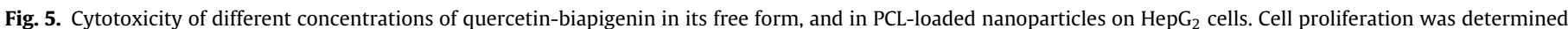

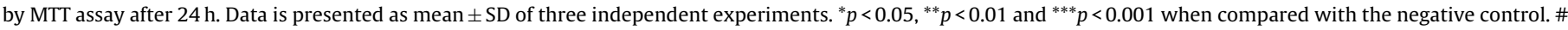
$p<0.05$, \#\# $p<0.01$ and \#\#\# $p<0.001$ when comparing two similar concentrations.

In the smaller concentrations tested free compounds are more effective, in terms of cell proliferation, than nanoparticles (significant for 5 and $10 \mu \mathrm{g} / \mathrm{mL}$, \# $p<0.05$ ).

Empty PCL nanoparticles revealed no significant interference in cell proliferation in the concentrations range tested (neither toxic nor protective activity) (data not shown).

Results show that nanoencapsulation of quercetin and biapigenin significantly reduced intrinsic toxicity of the compounds at high concentrations $(25,50$ and $100 \mu \mathrm{g} / \mathrm{mL}$ ). In contrast, with free compounds, cell viability is decreased in $30 \%$ at the concentration of $25 \mu \mathrm{g} / \mathrm{mL}$ and about $80 \%$ at $100 \mu \mathrm{g} / \mathrm{mL}$. These results, regarding quercetin toxicity, are coherent with others studies, in $\mathrm{HepG}_{2}$ and other cell lines [39,40-42], suggesting an intrinsic, non-selective, compound toxicity. There are no reports, to our knowledge, of biapigenin toxicity. However, this possibility cannot be excluded, as biapigenin is a flavonoid, and its known that this group of compounds can act also as prooxidants, on a concentration dependent manner [43].

One of the aims of preparing nanoparticles is to minimize toxicity of the compounds they convey. This was successfully achieved for higher compounds concentrations (100-50 $\mu \mathrm{g} / \mathrm{mL}$; Fig. 5). Contrarily, in concentrations lower than $10 \mu \mathrm{g} / \mathrm{mL}$, free compounds seem to promote higher cell viability in comparison with its nanoparticulate equivalent. This can be explained by an incomplete release of quercetin-biapigenin from the nanoparticulate system, at $3 \mathrm{~h}$ time.

Particle cellular uptake can be affected by several factors, such as size and shape, cell line in study, nanoparticle composition and surface properties [44]. In this study, particle size and surface properties, namely charge, are considered to be determinants for cellular uptake efficiency. Taking in consideration that both cell membrane and quercetin-biapigenin PCL-loaded nanoparticles are negatively charged the repulsive forces between them could determine a lower cellular uptake from the PCL-loaded nanoparticles, in comparison with the compounds in its free form. Internalization of PCL-loaded nanoparticles, as described in other studies, is believed to occur through nonspecific binding and clustering of the particles on scattered cationic sites present on the plasma membrane, and consequent endocytosis [44].

\subsection{Protective effect of quercetin-biapigenin and} quercetin-biapigenin poly( $\epsilon$-caprolactone)-loaded nanoparticles against tert-butylhydroperoxide-induced toxicity

$t$-BOOH is a short chain analog of lipid hydroperoxides which mimics the toxic effect of peroxidized fatty acids. This prooxidant is considered a useful model for investigating the mechanism of oxidative cell injury. It can act by two distinct mechanisms. The first comprises the generation of peroxy radicals in cytosol through its interaction with ferrous iron in a reaction similar to the Fenton reaction or cytochrome P450, thus playing an important role in free radical formation, inducing oxidative DNA damage and lipid peroxidation. The second is dependent on the depletion of the reduced from of glutathione. $t-\mathrm{BOOH}$ can be metabolized by Se-glutathione peroxidase (GPx) with formation of glutathione disulfide (GSSG) from its reduced form reduced glutathione (GSH). These phenomena are similar to those related to cell and tissue oxidative stress. Depletion of GSH and nicotinamine adenine dinucleotide phosphate (reduced form) (NADPH) oxidation are associated with altered calcium homeostasis, and related with changes in mitochondrial permeability, accompanied by a depolarization of mitochondrial potential, which can lead to cell injury and death $[45,46]$.

Oxidant $t-\mathrm{BOOH}$ is considered a model compound that can be used to induce oxidative stress in cell systems by producing free radical intermediates, increase of which might be a primary factor for hepatotoxicity [48]. $\mathrm{HepG}_{2}$ cell line has been used in several studies to evaluate the toxicity or protective effects of several compounds against oxidative stress $t$-BOOH-induced [16,2048].

In the present study HepG2 cells were exposed to an oxidative insult induced by $t$-BOOH. Co-incubation of $\mathrm{HepG}_{2}$ cells with $t$-BOOH and quercetin-biapigenin or its polymeric nanoparticles reflects a direct antioxidant effect of the compounds on $t-\mathrm{BOOH}-$ induced cell toxicity. Two incubation times ( 3.5 and $5 \mathrm{~h}$ ) were selected in order to understand the compounds and nanoparticle activity on $\mathrm{HepG}_{2}$ cells.

Free compounds revealed better protective activity than the correspondent nanoparticles in all the concentrations tested (Figs. 6 and 7). In the second co-incubation time selected, $5 \mathrm{~h}$, there is an even smaller activity by quercetin-biapigenin PCL-loaded 


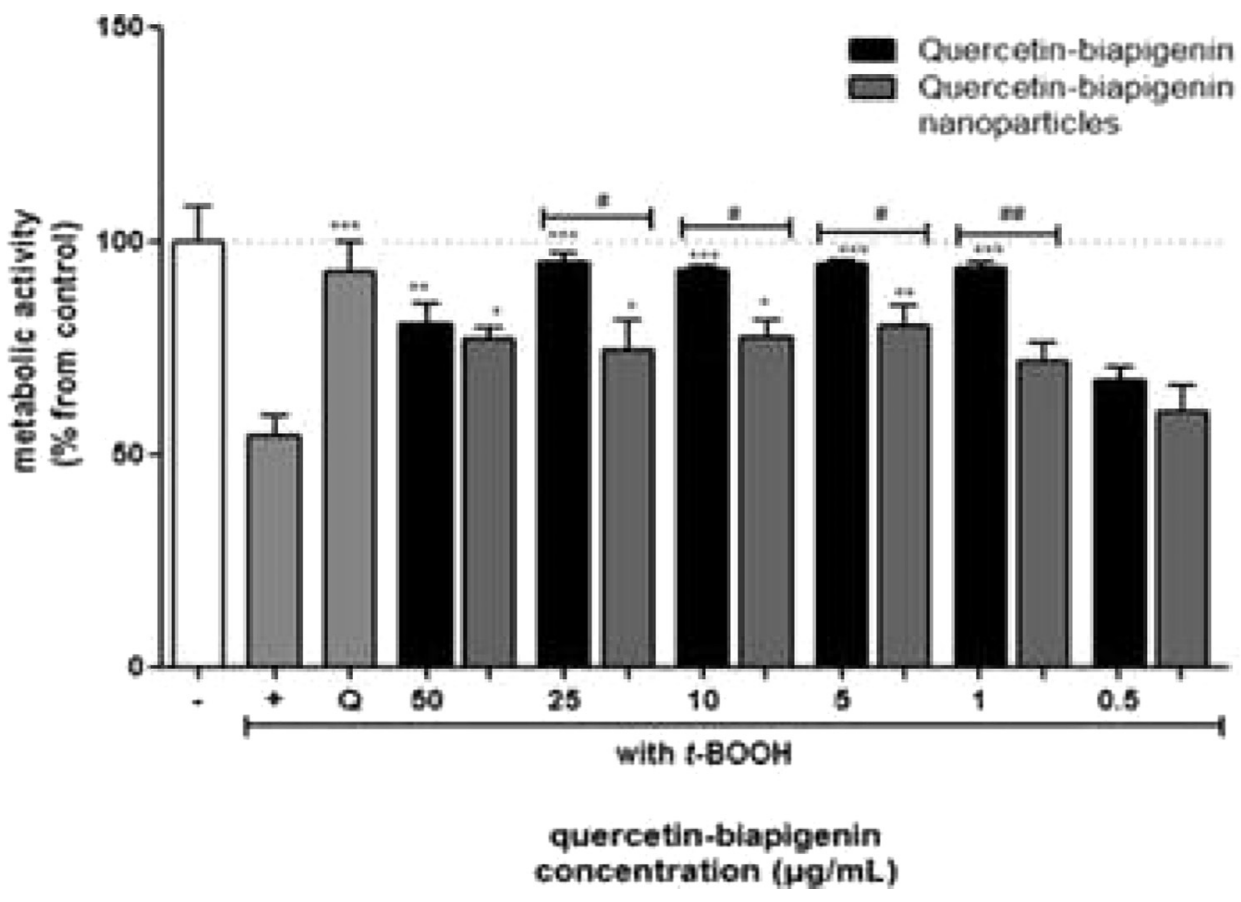

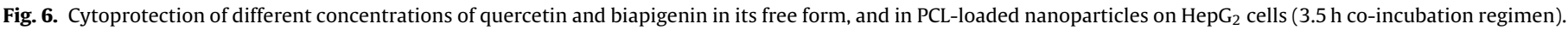

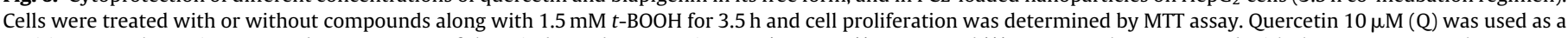

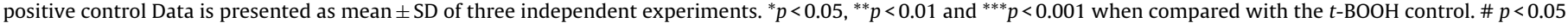
and \#\# $p<0.01$ when comparing two similar concentrations.

nanoparticles, comparing with the first period of time, coherently with the justification for this incubation regimen. With an increase in time, from 3.5 to $5 \mathrm{~h}, t$-BOOH would supposedly enter even more in the cells becoming very difficult for the cells to protect themselves from that insult.
When exposing $\mathrm{HepG}_{2}$ cells simultaneously to quercetinbiapigenin or its PCL-loaded nanoparticles and $t$-BOOH for $3.5 \mathrm{~h}$ and with compound concentration between 1 and $25 \mu \mathrm{g} / \mathrm{mL}, t$ $\mathrm{BOOH}$-induced toxicity was inhibited almost completely, with cells metabolic activity values near to those of $t-\mathrm{BOOH}$-untreated cells. In these concentrations free quercetin-biapigenin were significantly

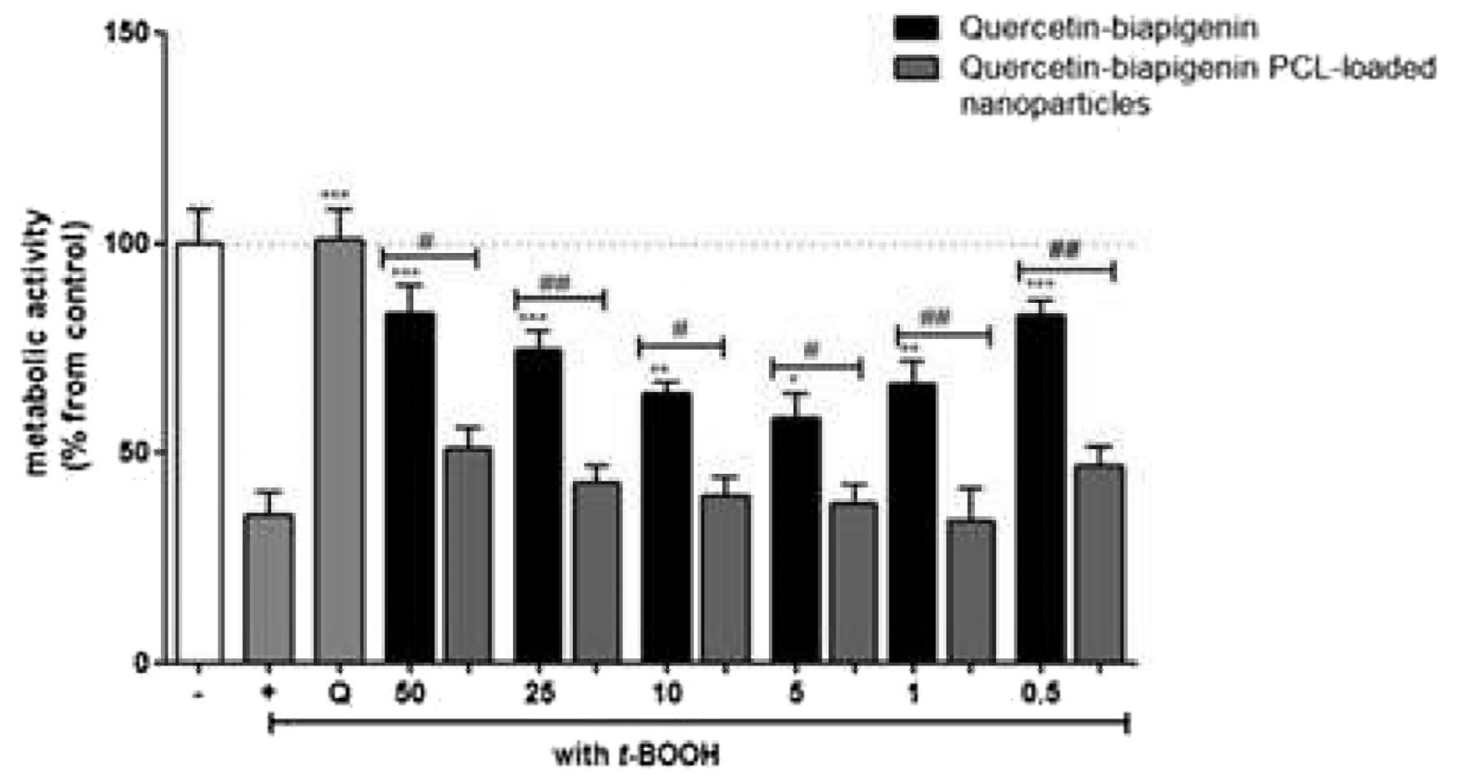

\section{quercetin-blapigenin concentration ( $\mathrm{\mu g} / \mathrm{mL}$ )}

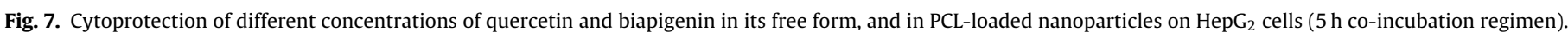

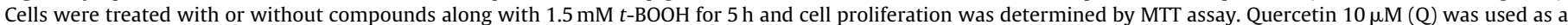

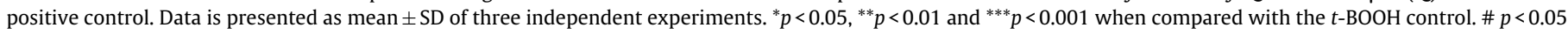
and \#\# $p<0.01$ when comparing two similar concentrations. 


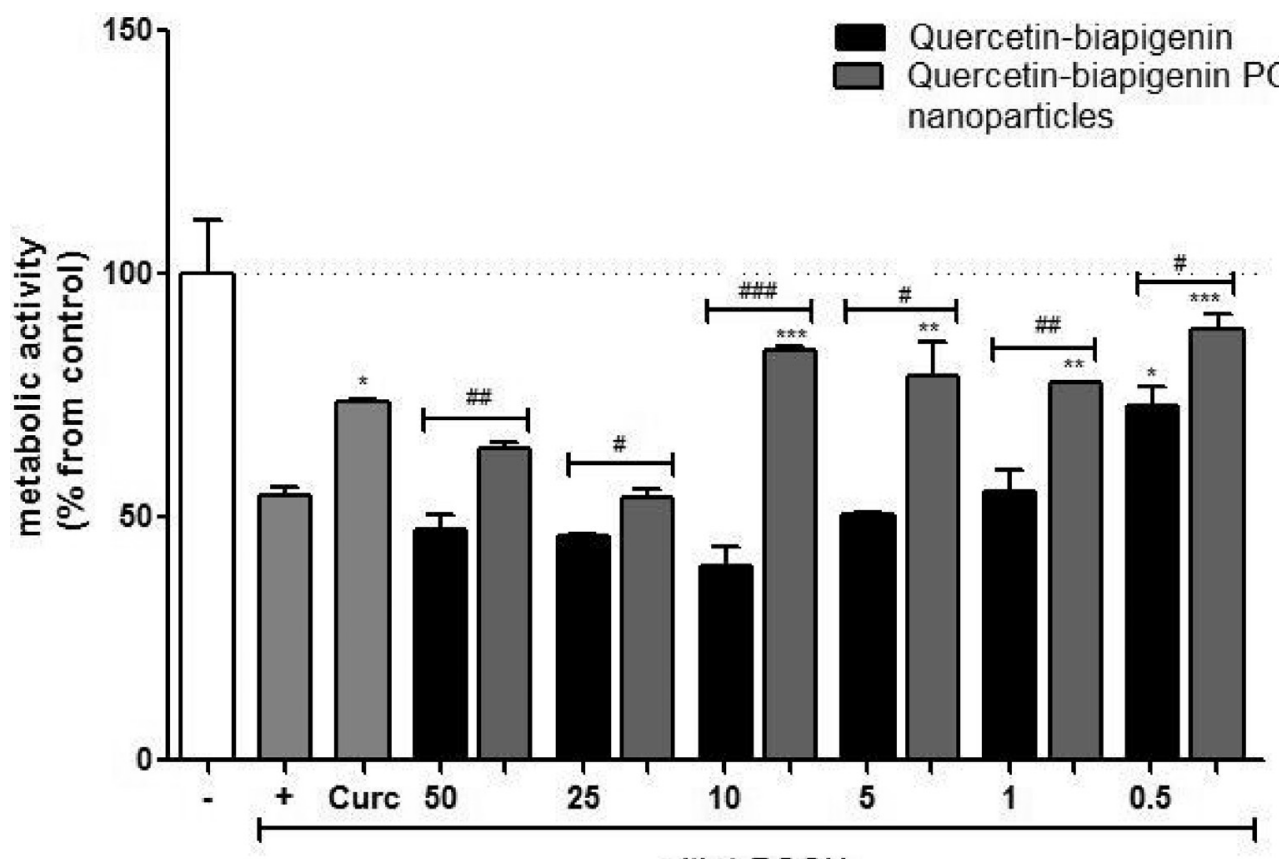

with $\mathrm{t}-\mathrm{BOOH}$

\author{
quercetin-biapigenin \\ concentration $(\mu \mathrm{g} / \mathrm{mL})$
}

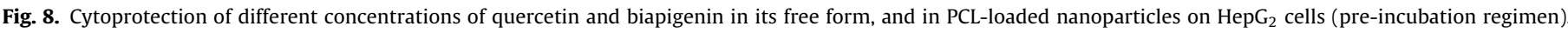

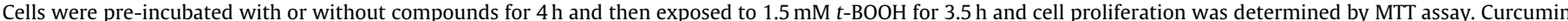

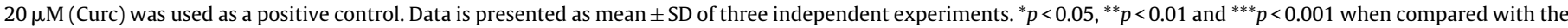
$t$-BOOH control. \# $p<0.05$, \#\# $p<0.01$ and \#\#\# $p<0.001$ when comparing two similar concentrations.

more protective than the corresponding polymeric nanoparticles $(p<0.01$ at $1 \mu \mathrm{g} / \mathrm{mL}$ and $p<0.05$ between 5 and $25 \mu \mathrm{g} / \mathrm{mL}$ ) (Fig. 6).

In the co-incubation regimen of $5 \mathrm{~h}$ exposure time, a decrease in cell viability was perceived in the group of cells exposed to $t$-BOOH and quercetin-biapigenin PCL-loaded nanoparticles, to values close to those of the toxic alone (Fig. 7). This is evidence of a non-protective activity of the nanoparticles. As for the compounds in its free form, as stated above, they had a significant protective activity against $t-\mathrm{BOOH}$, in all concentrations tested, in comparison to the nanoparticles. This could be explained by the higher compound/particle competition for proper cell uptake, leading to an advantage for the compounds with better internalization properties.

In the pre incubation regimen, $\mathrm{HepG}_{2}$ cells were first exposed to the compounds and its polymeric nanoparticles, followed by a recovery period with fresh medium before addition of oxidant $t$ $\mathrm{BOOH}$.

Pre-treating $\mathrm{HepG}_{2}$ cells with quercetin-biapigenin PCL-loaded nanoparticles revealed protective activity in all of the concentrations tested, in comparison with the compounds in its free form. When comparing with $t$-BOOH control group, quercetin-biapigenin PCL-loaded nanoparticles presented protective properties in concentrations bellow $10 \mu \mathrm{g} / \mathrm{mL}$ (Fig. 8). Here, cells toxicity was significantly decreased (in comparison with $t$-BOOH-untreated cells). The isolated compounds did not possess a significant protective effect on the cells (except in the lowest concentration tested), and an increase in cell proliferation in a dose-dependent manner was observed.

A smaller concentration $(0.5 \mu \mathrm{g} / \mathrm{mL})$ was added, on the pre- and co-incubation regimen assays, to better understand the activity of low compound concentrations. These assays were not performed with the higher concentration studied $(100 \mu \mathrm{g} / \mathrm{mL})$ due to significant toxicity of the compounds.

In the pre-incubation regimen, better results, in terms of cytoprotection, were obtained. This can be possibly justified by the intrinsic antioxidant properties of the compounds, which lead to an increase in cells protective mechanisms and their prooxidant activity as well as a possible higher internalization [23]. Deleterious activity of quercetin and biapigenin, such as other flavonoids, can be associated with toxicity, as previously described, but also as a potential protector. In this case, compounds would impose a mild oxidative stress to the cells, inducing antioxidant defenses and biotransformation enzymes, leading consequently to cytoprotection [43]. Results regarding quercetin and biapigenin in its free form could be justified by a more rapid metabolization (taking in consideration that these compounds, like other flavonoids, are considered xenobiotics, thus being rapidly metabolized) than the PCL-loaded nanoparticles, which would diminish the amount of compounds able to interact and protect the cells. The PCL-loaded nanoparticles, less toxic than the compounds in its free form, induce a higher protection. Here, the minor stress caused by the encapsulated compounds, once inside the cells, would lead to an increase of internal cell defenses, which would better protect against $t-\mathrm{BOOH}$ insult. These results, which are indicative that a small amount of compounds is enough to convey protective effects in $\mathrm{HepG}_{2}$ cells, are consistent with others [41].

\subsection{Lipid peroxidation}

Lipid peroxidation of polyunsaturated lipids in membranes is considered an important type of molecular lesion induced by free radicals [16]. During this process unsaturated lipids can be oxidized and cut into fragments, like alkanes, malondialdehyde (MDA) and 


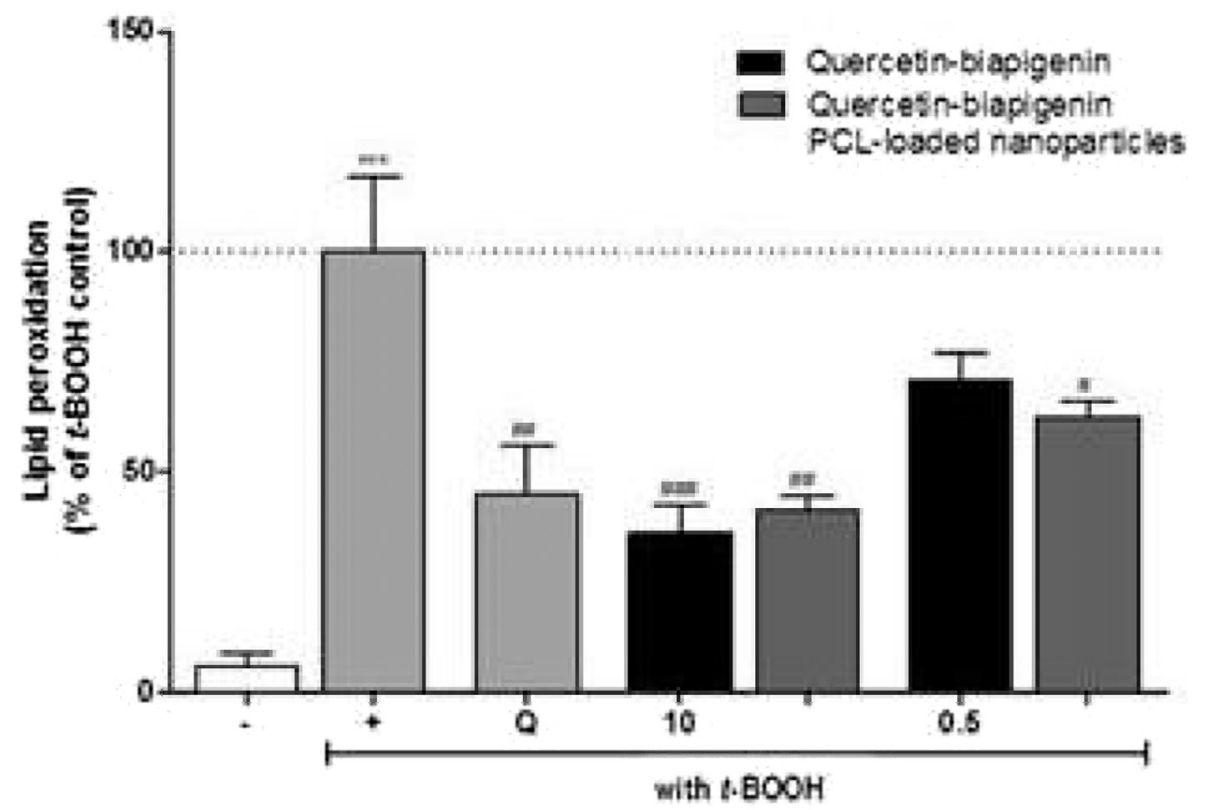

\section{quercetin-biapigenin concentration ( $\mu \mathrm{g} / \mathrm{mL})$}

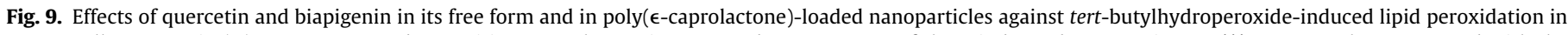

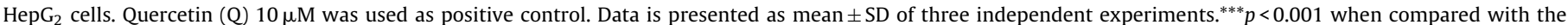
negative control. \# $p<0.05 \# \# p<0.01$ and \#\#\# $p<0.001$ when compared with the tert-butyl hydroperoxide control.

alkenals, chemically very reactive and, if not removed from the cell, able to cause cellular damage [16]. MDA has been widely used as an index of lipid peroxidation since it has been found to be elevated in several diseases thought to be related with free radical damage $[2,3]$.

Lipid peroxidation requires metal ions, like Fe(III) and Fe(II), that act as catalysts in redox reactions between oxygen and biological macromolecules. These ions facilitate, therefore, the conversion of superoxide anion and hydrogen peroxide to hydroxyl radical, a species proposed to initiate lipid peroxidation [49].

In order to study the effects of quercetin and biapigenin in its free form and its PCL-loaded nanoparticles against $t$-BOOHinduced lipid peroxidation, in a co-incubation regimen (with 3.5 and $5 \mathrm{~h}$ time) two concentrations were used: $\mathrm{i}-10 \mu \mathrm{g} / \mathrm{mL}$ that effectively protected against cell death in the range of $80-90 \%$ and, ii $-0.5 \mu \mathrm{g} / \mathrm{mL}$, which did not reveal significant protective activity. As shown in Fig. 9, $t$-BOOH-induced lipid peroxidation was significantly decreased, (approximately $60 \%$ ) with quercetin-biapigenin $10 \mu \mathrm{g} / \mathrm{mL}$. Only PCL-loaded nanoparticles with a compound concentration of $0.5 \mu \mathrm{g} / \mathrm{mL}$ significantly inhibit $t$-BOOH-induced lipid peroxidation in $\mathrm{HepG}_{2}$ cells $(p<0.05$, when compared with $t$ $\mathrm{BOOH}$-treated cells). These results are coherent with those of the co-incubation MTT assay.

In this study $t$-BOOH-induced lipid peroxidation in HepG2 cells was attenuated in all the tested scenarios, exception made with the co-incubation of $t$-BOOH and quercetin-biapigenin $0.5 \mu \mathrm{g} / \mathrm{mL}$. The inhibition was more intense when using quercetin-biapigenin or its PCL-loaded nanoparticles with compounds concentration of $10 \mu \mathrm{g} / \mathrm{mL}$, coherently with those of the co-incubation MTT assay. There seems to exist, therefore, a correlation between the cytoprotection and the prevention of lipid peroxidation in $\mathrm{HepG}_{2}$ cells. The ability that quercetin and possibly biapigenin have to chelate metal ions, specifically iron (II) can explain the results obtained in this study. Moreover biapigenin presented a better iron-mediated lipid peroxidation inhibition potential than quercetin [21]. The molecu- lar conformation of biapigenin, considering that it can insert itself in the cellular membrane [6] can also help to explain these results.

\section{Conclusions}

A purified fraction containing a mixture of quercetin and biapigenin, two antioxidant and neuroprotective compounds, was isolated from $H$. perforatum. The mixture was successfully encapsulated into PCL nanoparticles using solvent displacement method, resulting in nanoparticles ranging from 185 to $250 \mathrm{~nm}$ in size. The better proportion of PCL:compounds of those studied was of 1:0.1, with an $\mathrm{AE}$ of $99.7 \%$ and a loading capacity of $5.3 \%$. As for the release profile, a biphasic release was verified, including an initial burst effect followed by a slow, constant release. As to our knowledge, this is the first report of the encapsulation of a quercetin and biapigenin mixture in a polymeric matrix, specifically, in PCL.

Antioxidant activity assays revealed that the functional properties of the compounds were maintained after encapsulation. High encapsulation efficiency, small size, a sustained release and the reproducibility of the production method make quercetinbiapigenin loaded PCL nanoparticles a suitable candidate for further studies. The hepatoprotective activity of quercetin-biapigenin and its PCL-loaded nanoparticles was also studied. PCL-loaded nanoparticles protect $\mathrm{HepG}_{2}$ cells from intrinsic quercetin and biapigenin toxicity at high concentrations. In lower concentrations compounds in its free form were more effective. Depending on the incubation regimen, regarding $t$ - $\mathrm{BOOH}$-induced toxicity, quercetin-biapigenin PCL-loaded nanoparticles (pre-incubation) or compounds in its free form (co-incubation) were more effective in protecting $\mathrm{HepG}_{2}$ cells. Differences can be explained by cell uptake and competitive factors, respectively.

Taking also in consideration quercetin and biapigenin antioxidant and neuroprotective properties, it will be relevant to investigate the potential of the nanoparticles described in this study to reach the brain-blood barrier and the brain. This nanoparticu- 
late system might be used in the treatment of neurodegenerative diseases, such as Alzheimer and Parkinson disease.

\section{Acknowledgments}

The authors would like to thank Rui Fernandes, from I3S and IBMC, for TEM analysis. This work was supported by Fundação para a Ciência e Tecnologia (FCT, Portugal), projects PTDC/AGRALI/105169/2008, PEst-OE/AGR/UI4033/2014. Ana Isabel Oliveira was supported by Escola Superior de Tecnologia da Saúde do Porto and Instituto Politécnico do Porto (Programa de Formação Avançada de Docentes).

\section{References}

[1] L.A. Pham-Huy, H. He, C. Pham-Huy, Free radicals: antioxidants in disease and health, Int. J. Biomedicam Sci. 4 (2) (2008) 89-96.

[2] A.I. Oliveira, C. Pinho, B. Sarmento, A.C.P. Dias, Neuroprotective activity of hypericum perforatum and its major components, Front. Plant Sci. 7 (2016) 1004

[3] B.A. Silva, P.J. Oliveira, A.C.P. Dias, J.O. Malva, Mitochondria as targets for neuronal protection against excitotoxicity: a role for phenolic compounds? Cent. Nerv. Syst. Agents Med. Chem. 7 (2007) 205-222.

[4] C. Behl, Alzheimer's disease and oxidative stress: implications for novel therapeutic approaches, Prog. Neurobiol. 57 (3) (1999) 301-323.

[5] M. Wurglics, M. Schubert-Zsilavecz, Hypericum perforatum: a 'modern' herbal antidepressant: pharmacokinetics of active ingredients, Clin. Pharmacokinet. 45 (5) (2006) 449-468.

[6] B. Silva, P.J. Oliveira, A. Dias, J.O. Malva, Quercetin, kaempferol and biapigenin from Hypericum perforatum are neuroprotective against excitotoxic insults, Neurotox. Res. 13 (3-4) (2008) 265-279.

[7] B.A. Silva, F. Ferreres, J.O. Malva, A.C.P. Dias, Phytochemical and antioxidant characterization of Hypericum perforatum alcoholic extracts, Food Chem. 90 (2005) 157-167.

[8] B.A. Silva, A.C.P. Dias, F. Ferreres, J.O. Malva, C. Oliveira, Neuroprotective effect of $H$. perforatum extracts on $\beta$-Amyloid-induced neurotoxicity, Neurotox. Res. 6 (2) (2004) 119-130.

[9] B.A. Silva, P.J. Oliveira, A. Cristóvão, A.C.P. Dias, J.O. Malva, Biapigenin modulates the activity of the adenine nucleotide translocase in isolated rat brain mitochondria, Neurotox. Res. 17 (1) (2010) 75-90.

[10] F. Dajas, Life or death: neuroprotective and anticancer effects of quercetin, J. Ethnopharmacol. 143 (2) (2012) 383-396.

[11] N. Suematsu, M. Hosoda, K. Fujimori, Protective effects of quercetin against hydrogen peroxide-induced apoptosis in human neuronal SH-SY5 Y cells, Neurosci. Lett. 504 (3) (2011) 223-227.

[12] T. Pralhad, K. Rajendrakumar, Study of freeze-dried quercetin-cyclodextrin binary systems by DSC, FT-IR, X-ray diffraction and SEM analysis, J. Pharm. Biomed. Anal. 34 (2) (2004) 333-339.

[13] Y.J. Moon, L. Wang, R. DiCenzo, M.E. Morris, Quercetin pharmacokinetics in humans, Biopharm. Drig Dispos. 29 (2008) 205-217.

[14] E. Barbu, E. Molnar, J. Tsibouklis, D.C. Gorecki, The potential for nanoparticle-based drug delivery to the brain: overcoming the blood-brain barrier, Expert Opin. Drug Deliv. 6 (6) (2009) 553-565.

[15] A. Kumari, S.K. Yadav, S.C. Yadav, Biodegradable polymeric nanoparticles based drug delivery systems, Colloids Surf. B Biointerfaces 75 (1) (2010) 1-18.

[16] C.F. Lima, M. Fernandes-Ferreira, C. Pereira-Wilson, Phenolic compounds protect HepG2 cells from oxidative damage: relevance of glutathione levels, Life Sci. 79 (21) (2006) 2056-2068.

[17] S. Knasmuller, V. Mersch-Sundermann, S. Kevekordes, F. Darroudi, W.W. Huber, C. Hoelzl, J. Bichler, B.J. Majer, Use of human-derived liver cell lines for the detection of environmental and dietary genotoxicants; current state of knowledge, Toxicology 198 (1-3) (2004) 315-328.

[18] A.C.P. Dias, R.M. Seabra, P.B. Andrade, M. Fernandes-Ferreira, The development and evaluation of an HPLC-DAD method for the analysis of the phenolic fractions from in vivo and in vitro biomass of Hypericum species, J. Liq. Chromatogr. Relat. Technol. 22 (2) (1999) 215-227.

[19] G. Marslin, B. Sarmento, F. Gregory, J.A.R. Martins, C.J.R. Silva, A. Gomes, M.P Sárria, O. Coutinho, A.C.P. Dias, Curcumin Encapsulated into Methoxy Poly(Ethylene Glycol) Poly( $\varepsilon$-Caprolactone) Nanoparticles Increases Cellular Uptake and Neuroprotective Effect in Glioma Cells, Planta Med. 83 (05) (2017) 434-444.

[20] C.F. Lima, P.B. Andrade, R.M. Seabra, M. Fernandes-Ferreira, C. Pereira-Wilson, The drinking of a Salvia officinalis infusion improves liver antioxidant status in mice and rats, J. Ethnopharmacol. 97 (2) (2005) 383-389.

[21] A. Kumaran, R.J. karunakaran, Antioxidant and free radical scavenging activity of an aqueous extract of Coleus aromaticus, Food Chem. 97 (1) (2006) 109-114.

[22] A.C. Carvalho, F. Gregory, A.C.P. Dias, C.F. Lima, Methanolic extract of Hypericum perforatum cells elicited with Agrobacterium tumefaciens provides protection against oxidative stress induced in human HepG2 cells, Ind. Crops Prod. 59 (2014) 177-183.

[23] C. Vauthier, K. Bouchemal, Methods for the preparation and manufacture of polymeric nanoparticles, Pharm. Res. 26 (5) (2009) 1025-1058.

[24] H. Fessi, J. Puisieux, J.Ph. Devissaguet, N. Ammoury, S. Benita, Nanocapsule formation by interfacial polymer deposition following solvent displacement, Int. J. Pharm. 55 (1989) R1-R4.

[25] V.J. Mohanraj, Y.N. Chen, Nanoparticles - a review, Trop. J. Pharm. Res. 5 (1) (2006) 561-573.

[26] Y. Hu, X. Jiang, Y. Ding, L. Zhang, C. Yang, J. Zhang, J. Chen, Y. Yang, Preparation and drug release behaviors of nimodipine-loaded poly(caprolactone)-poly(ethylene oxide)-polylactide amphiphilic copolymer nanoparticles, Biomaterials 24 (13) (2003) 2395-2404.

[27] J.S. Chawla, M.M. Amiji, Biodegradable poly(epsilon-caprolctone) nanoparticle for tumor-targeted delivery of tamoxifen, Int. J. Pharm. 249 (1-2) (2002) 127-138

[28] K.S. Soppimath, T.M. Aminabhavi, A.R. Kulkarni, W.E. Rudzinski, Biodegradable polymeric nanoparticles as drug delivery devices, J. Control. Release 70 (1-2) (2001) 1-20.

[29] A. Kumari, S.K. Yadav, Y.B. Pakade, B. Singh, S.C. Yadav, Development of biodegradable nanoparticles for delivery of quercetin, Colloids Surf. B Biointerfaces 80 (2) (2010) 184-192.

[30] F. Alexis, S.S. Venkatraman, S.K. Rath, F. Boey, In vitro study of release mechanisms of paclitaxel and rapamycin from drug-incorporated biodegradable stent matrices, J. Control. Release 98 (1) (2004) 67-74.

[31] V.R. Sinha, K. Bansal, R. Kaushik, R. Kumria, A. Trehan, Poly-epsilon-caprolactone microspheres and nanospheres: an overview, Int. J. Pharm. 278 (1) (2004) 1-23.

[32] R. Vrijsen, L. Everaert, A. Boeyé, Antiviral activity of flavones and potentiation by ascorbate, J. Gen. Virol. 69 (1988) 1749-1751, http://dx.doi.org/10.1099/ 0022-1317-69-7-1749.

[33] I.G. Zenkevich, A.Y. Eshchenko, S.V. Makarova, A.G. Vitenberg, Y.G. Dobryakov, V.A. Utsal, Identification of the products of oxidation of quercetin by air oxygen at ambient temperature, Molecules 12 (3) (2007) 654-672.

[34] D.P. Makris, J.T. Rossiter, Heat-induced: metal-catalyzed oxidative degradation of quercetin and rutin (Quercetin 3-O-rhamnosylglucoside) in aqueous model systems, J. Agric. Food Chem. 48 (9) (2000) 3830-3838.

[35] T.H. Wu, F.L. Yen, L.T. Lin, T.R. Tsai, C.C. Lin, T.M. Cham, Preparation, physicochemical characterization, and antioxidant effects of quercetin nanoparticles, Int. J. Pharm. 346 (1-2) (2008) 160-168.

[36] L.M. Magalhaes, M.A. Segundo, S. Reis, J.L. Lima, Methodological aspects about in vitro evaluation of antioxidant properties, Anal. Chim. Acta 613 (1) (2008) $1-19$.

[37] M. Kessler, G. Ubeaud, L. Jung, Anti- and pro-oxidant activity of rutin and quercetin derivatives, J. Pharm. Pharmacol. 55 (1) (2003) 131-142.

[38] M. Leopoldini, N. Russo, S. Chiodo, M. Toscano, Iron chelation by the powerful antioxidant flavonoid quercetin, J. Agric. Food Chem. 54 (17) (2006) 6343-6351

[39] S.A. van Acker, G.P. van Balen, D.J. van den Berg, A. Bast, W.J. van der Vijgh, Influence of iron chelation on the antioxidant activity of flavonoids, Biochem. Pharmacol. 56 (8) (1998) 935-943.

[40] S. Wilkening, F. Stahl, A. Bader, Comparison of primary human hepatocytes and hepatoma cell line Hepg2 with regard to their biotransformation properties, Drug Metab. Dispos. 31 (8) (2003) 1035-1042.

[41] M. Alia, R. Mateos, S. Ramos, E. Lecumberri, L. Bravo, L. Goya, Influence of quercetin and rutin on growth and antioxidant defense system of a human hepatoma cell line (HepG2), Eur. J. Nutr. 45 (1) (2006) 19-28.

[42] F. Arredondo, C. Echeverry, J.A. Abin-Carriquiry, F. Blasina, K. Antunez, D.P. Jones, Y.M. Go, Y.L. Liang, F. Dajas, After cellular internalization, quercetin causes Nrf2 nuclear translocation, increases glutathione levels, and prevents neuronal death against an oxidative insult, Free Radic. Biol. Med. 49 (5) (2010) $738-747$.

[43] D. Prochazkova, I. Bousova, N. Wilhelmova, Antioxidant and prooxidant properties of flavonoids, Fitoterapia 82 (4) (2011) 513-523.

[44] A. Verma, F. Stellacci, Effect of surface properties on nanoparticle-cell interactions, Small 6 (1) (2010) 12-21.

[45] M.C. Lazze, R. Pizzala, M. Savio, L.A. Stivala, E. Prosperi, L. Bianchi, Anthocyanins protect against DNA damage induced by tert-butyl-hydroperoxide in rat smooth muscle and hepatoma cells, Mutat. Res./Fundam. Mol. Mech. Mutagen. 535 (1) (2003) 103-115.

[46] J.P. Piret, T. Arnould, B. Fuks, P. Chatelain, J. Remacle, C. Michiels, Mitochondria permeability transition-dependent tert-butyl hydroperoxide-induced apoptosis in hepatoma HepG2 cells, Biochem. Pharmacol. 67 (4) (2004) 611-620.

[47] B.A. Silva, J.O. Malva, A.C.P. Dias, Hypericum perforatum extracts and isolated phenolic compounds are effective antioxidants in several in vitro models of oxidative stress, Food Chem. 110 (2008) 611-619.

[48] J.H. Sohn, K.L. Han, S.H. Lee, J.K. Hwang, Protective effects of panduratin A against oxidative damage of tert-butylhydroperoxide in human HepG2 cells, Biol. Pharm. Bull. 28 (6) (2006) 1083-1086.

[49] S.J. Stohs, D. Bagchi, Oxidative mechanisms in the toxicity of metal ions, Free Radic. Biol. Med. 18 (2) (1995) 321-336. 